BMJ Health \& Care Informatics

\title{
Impact of DSMES app interventions on medication adherence in type 2 diabetes mellitus: systematic review and meta-analysis
}

\author{
Dumisani Enricho Nkhoma, ${ }^{1,2}$ Charles Jenya Soko, ${ }^{2}$ Kondwani Joseph Banda, ${ }^{3}$ \\ David Greenfield, ${ }^{4}$ Yu-Chuan (Jack) Li (D) , ${ }^{5,6,7,8,9}$ Usman lqbal (iD) ${ }^{2,6,10}$
}

To cite: Enricho Nkhoma D, Jenya Soko C, Joseph Banda $\mathrm{K}$, et al. Impact of DSMES app interventions on medication adherence in type 2 diabetes mellitus: systematic review and meta-analysis. BMJ Health Care Inform 2021;28:e100291. doi:10.1136/ bmjhci-2020-100291

- Additional supplemental material is published online only. To view, please visit the journal online (http://dx.doi.org/10. 1136/bmjhci-2020-100291).

Received 30 November 2020 Revised 18 February 2021 Accepted 15 March 2021
Check for updates

(C) Author(s) (or their employer(s)) 2021. Re-use permitted under CC BY-NC. No commercial re-use. See rights and permissions. Published by BMJ.

For numbered affiliations see end of article.

Correspondence to

Dr Usman Iqbal;

usmaniqbal@tmu.edu.tw

\section{ABSTRACT}

Objectives To conduct systematic review and metaanalysis of interventional studies to investigate the impact of diabetes self-management education and support (DSMES) apps on adherence in patients with type 2 diabetes mellitus (T2D).

Methods PubMed, Embase, CENTRAL, Web of Science, Scopus and ProQuest were searched, in addition to references of identified articles and similar reviews. Experimental studies, reported in English, assessing DSMES app intervention's impact on adherence and clinical outcomes of patients with T2D compared with usual care were included. Study bias was assessed using Cochrane Risk of Bias V.2.0 tool. Analysis plan involved narrative synthesis, moderator and meta-analysis.

Results Six randomised controlled trials were included, involving 696 participants (average age 57.6 years, SD 10.59). Mobile apps were mostly used for imputing clinical data, dietary intake or physical activity, and transmitting information to the provider. At 3 months, DSMES apps proved effective in improving medication adherence (standardized mean difference (SMD) $=0.393,95 \% \mathrm{Cl} 0.17$ to 0.61 ), glycated haemoglobin $(\mathrm{HbA} 1 \mathrm{c})$ (mean difference $(\mathrm{MD})=-0.314,95 \% \mathrm{Cl}-0.477$ to -0.151$)$ and Body Mass Index (BMI) (MD=-0.28, 95\% Cl -0.545 to -0.015$)$. All pooled estimates had low heterogeneity $(₹ 0 \%)$. Four studies had moderate risk of bias while one each was judged to be low and high risks, respectively.

Conclusion DSMES apps had significant small to moderate effects on medication adherence, $\mathrm{HbA1C}$ and BMI of patients with T2D compared with usual care. Apps were described as reliable, easy to use and convenient, though participants were required to be phone literate. Evidence comes from feasibility trials with generally moderate risk of bias. Larger trials with longer follow-up periods using theory-based interventions are required to improve current evidence.

\section{BACKGROUND}

The burden of diabetes mellitus (DM) has grown enormously over the years with its prevalence is estimated to be more than 500 million globally in 2018, being one of the leading causes of death worldwide with an estimated economic burden of around
US $\$ 1.3$ trillion as of $2015 .{ }^{1-3} \mathrm{DM}$ is characterised by two major types: type $1 \mathrm{DM}$ and type 2 diabetes mellitus (T2D), the latter constituting the majority of all known DM cases. Major risk factors for the disease include obesity and lack of exercise with long-term complications leading to stroke, leg amputation, and kidney and heart or eye problems. ${ }^{4}$ T2D has no cure and, as such, patients are bound to lifelong treatment of which, if committed to, is associated with prevention of complications, lower medical costs and consequently better quality of life. ${ }^{56}$

A commitment to T2D treatment means being fully adherent to medical prescriptions, diet and exercise plans. Treatment adherence is the extent to which a person's behaviour, medication use, and diet or lifestyle changes correspond to agreed recommendations from a healthcare provider. ${ }^{7}$ However, treatment adherence in patients with chronic diseases, including DM, has been reported to be suboptimal. ${ }^{89}$

Several techniques, such as single-dose regimens, reminders and easy packaging, have been used to improve treatment adherence. ${ }^{10}$ One of these techniques, diabetes self-management education and support (DSMES), has been used to cover lifestyle, medication, blood glucose monitoring and other psychosocial aspects of treatment-all of which have been associated with improved health outcomes and reduced medical costs. ${ }^{11}$ DSMES is a patient-centred and holistic approach that makes it one of the most ideal techniques to improve treatment adherence of patients with T2D.

Furthermore, delivery of DSMES through technologies such as mobile health (m-health) may improve coverage and convenience for patients. m-health helps to improve adherence including by setting automated reminders 
and messages, and simplifying tracking of medication and prescriptions. ${ }^{10}{ }^{12}$ It is the the most common technology adopted by WHO member states. ${ }^{13}$ In addition, the American Diabetes Association includes m-health into 'standard of medical care' for DM. ${ }^{14}$ Unfortunately despite this endorsement, m-health is often underused in managing DM. ${ }^{15}$ There is even lower patronage for apps that would help improve medication adherence. ${ }^{16}$

There is even fewer literature covering the effect of mobile app-delivered interventions that aim at improving adherence in the T2D population. Majority of prior reviews have concentrated on assessing effectiveness of SMS interventions on medication adherence. ${ }^{10} 17$ One review did report the effect of app-delivered interventions; however, the study population was heterogeneous with little representation of patients with T2D. ${ }^{18}$ Other reviews have aimed at assessing m-health intervention effects on glycaemic control. ${ }^{1920}$

However, to our knowledge, no review was found to evaluate impact of mobile app-delivered DSMES on adherence specifically in the T2D population. Studies have concentrated on medication adherence rather than lifestyle modification adherence. Furthermore, prior reviews have reported inconsistent findings, lack of clarity on definition and measurement of medication adherence, and inadequate use of theoretical frameworks in the study interventions. ${ }^{1021}$

Results of the current review would help add clarity to existing literature and offer quantitative evidence on the impact of app-delivered diabetes education and selfmanagement support on treatment adherence of patients with T2D. The review's findings would also offer crucial applicability details to information technology and health professionals involved in efforts to reduce global DM burden.

Thus, we aimed to assess the effectiveness of mobile app-delivered DSMES to improve medication adherence and clinical outcomes in patients with T2D. We conducted a systematic review and meta-analysis of experimental studies to address this goal.

\section{METHODS}

\section{Design and data sources}

We followed the Preferred Reporting Items for Systematic Reviews and Meta-Analyses (PRISMA). ${ }^{22}$ The study protocol was registered with Open Science Framework (link: https:/ / osf.io/z6sgk). Research team members (DEN and CJS) searched five academic databases from 1 to 14 August 2020, in two phases, for articles published from inception until 31 July 2020: PubMed, Embase, CENTRAL, Web of Science, ProQuest and Scopus. Medical Subject Headings (MeSH) and free-text terms were both used to combine keywords, as illustrated in the online supplemental file tables S1).

\section{ELIGIBILITY CRITERIA}

Studies were included if they had (1) adults, 18 years or older, with T2D; (2) independent analysis of participants with T2D in multidisease studies; (3) reported use of mobile apps giving DSMES as a principal intervention; (4) medication or lifestyle therapy adherence as an outcome; (5) experimental design; and (6) been reported in English. Studies were excluded if control groups used any type of digital technology or did not include routine diabetes care.

\section{Study collection, selection and data extraction}

The studies were independently screened (DEN and CJS) with disputes being resolved through consensus with a separate expert reviewer (UI). Variable and characteristics were independently extracted team members (DEN, CJS and KJB) using a standard sheet adopted from a Cochrane Public Health Group template. ${ }^{23}$ Missing information was sought after by contacting authors through email. If the data could still not be retrieved, the study was excluded from the quantitative analysis.

\section{QUALITY ASSESSMENT}

Study bias was assessed using Cochrane's Risk of Bias V.2.0 tool independently by team members (DEN and $\mathrm{KJB}) .{ }^{24}$ Studies were graded according to their randomisation process, deviation from intended interventions, handling of missing data, measurement of outcomes and reporting of results. Each domain was judged as high, low or moderate risk (some concerns). Overall risk of bias depended on the assessment of the individual domains. Assessment was to be conducted for each outcome. However, outcomes were grouped into primary (adherence) and clinical outcomes (glycated haemoglobin (HbA1c), Body Mass Index (BMI), blood pressure (BP) and total cholesterol). Across-study bias was assessed using a funnel plot and Egger's regression test.

\section{Data analysis}

Narrative synthesis

Qualitative analysis involved description of population and intervention characteristics. Items included acceptability, challenges and use of cointerventions.

\section{Quantitative analysis}

Fixed-effects meta-analysis was performed using pairwise comparison at 3 months after baseline measurements. Medication and patient adherence were presented using standardized mean difference (SMD) with 95\% CIs. Change in HbAlc, BMI, total cholesterol and BP would be expressed using difference in means (MD) with 95\% CI. Quantitative analysis was conducted using Comprehensive Meta-Analysis V.3 (Biostat, Englewood, USA). During analysis, an outcome, patient adherence, was created as an aggregate of medication and lifestyle adherence estimates. Preintervention and postintervention score correlation was set at 0.5 . For studies presenting results as median and IQR or range, their data were converted into mean and $\mathrm{SD} .{ }^{25}{ }^{26}$ SDs were also calculated from $95 \%$ CIs. ${ }^{27}$ 


\section{Heterogeneity}

Heterogeneity was assessed using the $I^{2}$ statistic. It was expressed as low (less than 25\%), moderate (26\%-74\%) or high (more than $75 \%$ ), ${ }^{28}$ and importantly, interpreted according to its general context. Further explanations of high heterogeneity would be done in moderator analysis.

\section{Moderator analysis}

Subgroup analysis aimed to assess measures of effect according to intervention characteristics such as presence of cointervention, theoretical frameworks, content (diabetes self-management education/diabetes selfmanagement support/DSMES), risk of bias assessment presence of comorbidities or complications and frequency (daily/weekly/monthly). We expected to do

metaregression using the following variables: age, gender ratio, sample size, duration of disease and mean HbA1c, BP and BMI levels.

\section{Sensitivity analysis}

We aimed to assess the robustness of review estimates. This was performed by changing of preintervention and postintervention value correlation from 0.5 to 0.2 and 0.8 , respectively.

\section{RESULTS}

A total of 3460 articles were identified, from which 405 were duplicates or published in languages other than English. A total of 3080 articles were screened for eligibility. After screening using title and abstracts, 27 articles underwent full-text review from which 21 studies were excluded due to ineligible population, intervention, study design or outcome. Finally, six studies were included into the review, one of which was excluded from quantitative analysis due to insufficient data. ${ }^{29}$ All studies were conducted in Asia and Europe. Further details are illustrated in figure 1.

Study characteristics were assessed for diabetes duration, participant age and gender ratio, comorbidities and medications (table 1 ). The review population consisted of 696 participants with an average age of 57.6 years (SD 10.59), and 320 (45.98\%) were female. Duration of disease since diagnosis ranged from 6 months to 18 years, with all studies reporting presence of concurrent hypertension and dyslipidaemia in varying percentages. One study reported presence of complications such as neuropathy and kidney disease. ${ }^{30}$

Studies were randomised controlled trials (RCTs) with either as a single-blind or open-label trial, and all, except one, ${ }^{31}$ were exploratory. One study was conducted in a tertiary clinic, ${ }^{30}$ while the rest were conducted in primary healthcare (PHC) settings.

Interventions were used daily with the implementation period ranging from 6 weeks to 6 months. Two studies offered both self-management support and education. ${ }^{29} 32$ The rest offered only self-management support. Use of theoretical framework was only reported in one study. ${ }^{33}$ Compliance to mobile app interventions ranged from $69.6 \%$ to $88.0 \%$.

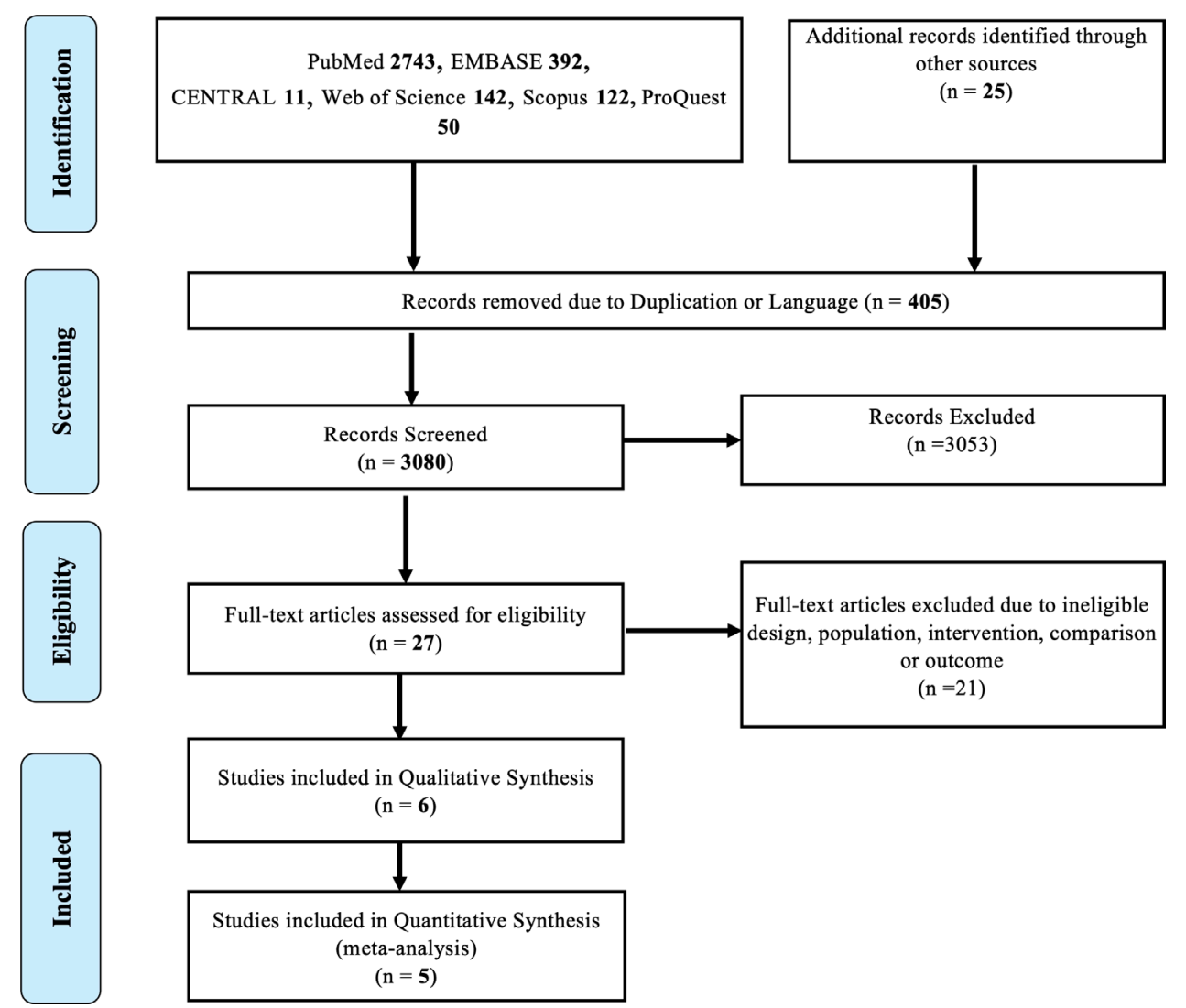

Figure 1 Research framework according to Preferred Reporting Items for Systematic Reviews and Meta-Analyses guidelines. 
Table 1 Characteristics of included studies and interventions

\begin{tabular}{llll}
\hline ID & Population & Design & Intervention \\
\hline ID, country & 1. Diabetes duration. & 1. Setting. & 1. Functions. \\
& 2. Age. & 2. Sample size. & 2. Cointerventions. \\
& 3. Female ratio. & 3. Intervention & 3. Theory. \\
& 4. Comorbidities. & duration. & 4. DSME/DSMES. \\
5. Medication. & 4. Blinding. & \\
& & 5. Design. & \\
& & 6. Control. & \\
Brath et al, ${ }^{34}$ & 1. ROB. & 1. Received data from e-blister and transmitted to \\
Austria & 2. 69.4 (SD 4.8). & 1. PHC. I=53, C=53. & providers' server. \\
& 3. $30(54.5 \%)$. & 3. 6 months. & 2. e-blister, provider portal and reminder calls. \\
& 4. HTN and HCHL. & 4. Single. & 3. NR. \\
& 5. Oral AHs. & 5. Crossover. & 4. DSMS.
\end{tabular}

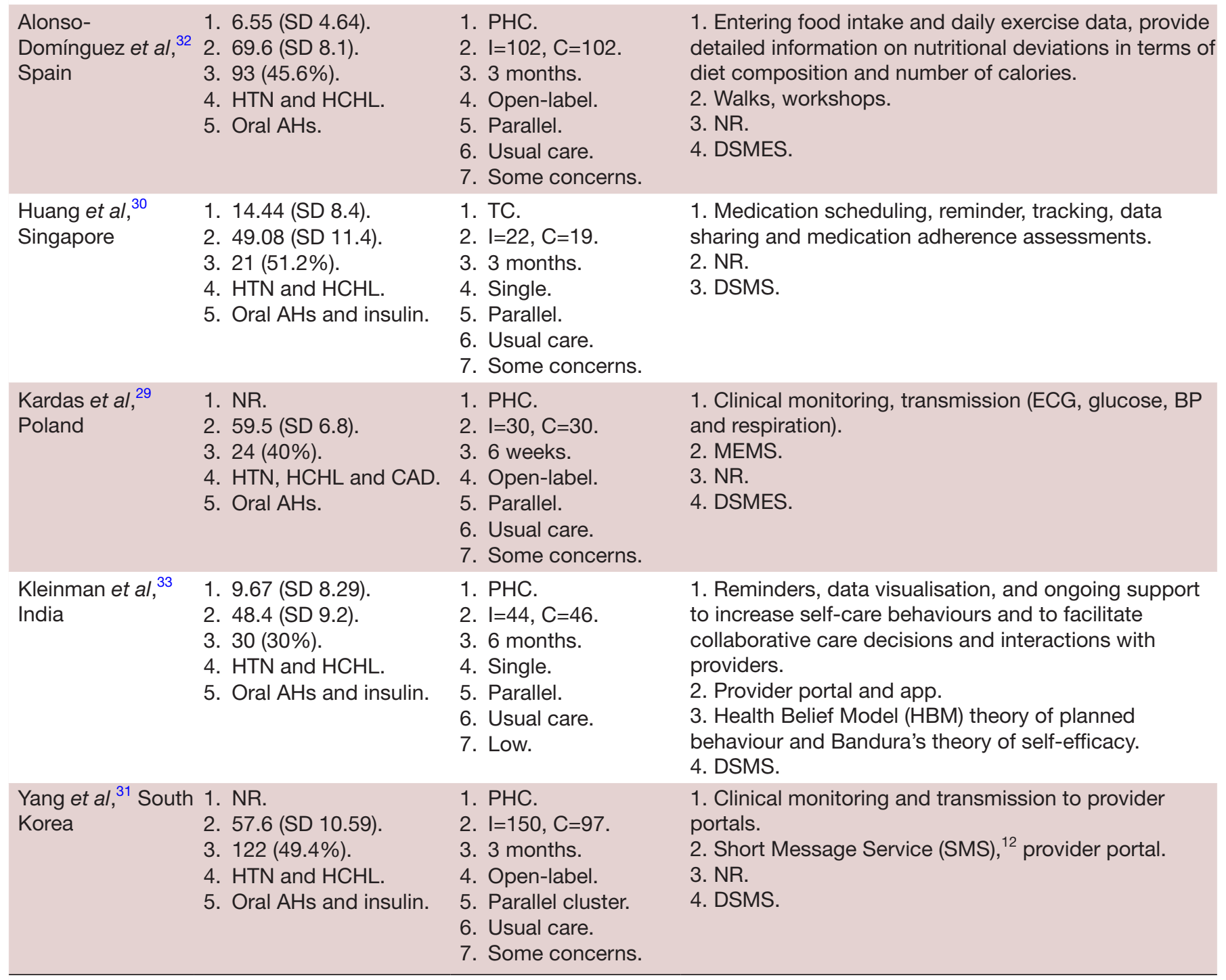

Comorbidities: HTN, HCHL and CAD.

Settings: PHC and TC.

Sample size: I and C.

$\mathrm{AH}$, antihyperglycaemic; BP, blood pressure; C, control group; CAD, coronary artery disease; DSMES, diabetes self-management education and support; DSMS, diabetes self-management support; HBM, Health Belief Model; HCHL, hypercholesterolaemia; HTN, hypertension; I, intervention group; MEMS, Medication Event Monitoring System; NR, not reported; PHC, primary healthcare; ROB, risk of bias; SMS, Short Message Service; TC, tertiary care. 
Mobile app functions included recording food intake, physical activity and clinical information such as BP and glucose levels. ${ }^{29-34}$ Other functions included alerting users if their uploaded data deviated from prespecified standards. ${ }^{30} 32$ Intervention apps also helped participants track and schedule medication, visualise and transmit data to providers and act as automated reminders. ${ }^{29-34}$ For measurement of clinical data, participants were equipped with instruments such as glucose monitors and strips.

The most common cointervention was provider portals which helped providers review transmitted patient data which they used to offer feedback to participants. ${ }^{31} 3334$ Additional reminders were provided in two studies. ${ }^{31} 34$ Brath $e t a l^{34}$ offered e-blisters, which helped participants adhere to their medication. ${ }^{34}$ Most interventions were guided by physicians. Control groups were offered usual clinic consultations, except for one study in which participants also received standard blisters and medication diaries. ${ }^{34}$

Acceptability was high among participants. Participants commended apps for their speed, ease of use and convenience. ${ }^{29} 3034$ Other participants reported that they liked that providers were familiar with their regimens. ${ }^{34}$ Participants indicated that interventions would improve their self-confidence in managing diseases and that they would use app afterwards. ${ }^{29} 30$
In terms of operation ability, participants were expected to have a working knowledge of phones and apps. In addition, researchers offered training to all participants to ensure proper use of interventions. Some of the challenges reported included technical complexity, 34 frequent need to recharge gadgets and lack of glucose strips. $^{29}$

\section{Quality of studies}

In terms of risk of bias, two studies were deemed as high $^{34}$ and low risks. ${ }^{33}$ The rest were judged as having some concerns. ${ }^{29-32}$ Major concerns were inadequate reporting of randomisation process, ${ }^{29-31} 34$ possibility of reporting bias, ${ }^{30-32}$ lack of blinding ${ }^{30-32} 34$ and lack of optimal handling of missing data. ${ }^{30} 3134$ Risk of bias assessment is illustrated in figure 2. Attrition rates ranged from $0 \%$ to $31 \%$. Publication bias was not detected $(\mathrm{p}=0.398)$.

\section{Moderator analysis and sensitivity analysis}

Due to the small number of studies, subgroup analyses were not performed. Changing precorrelation/postcorrelation from 0.5 to 0.2 and 0.8 did not significantly alter review effect estimates (see online supplemental figures S2-7).

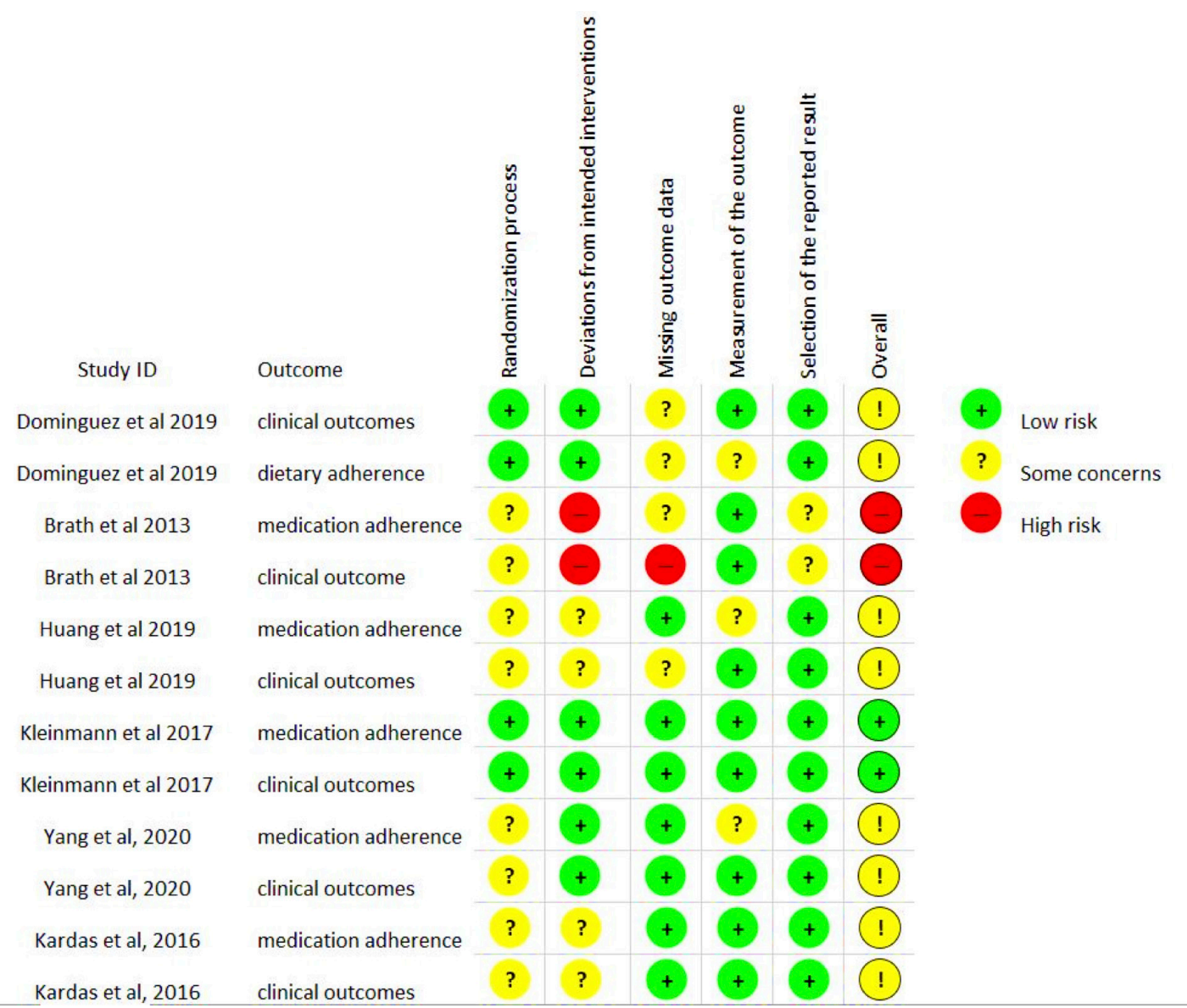

Figure 2 Risk of bias assessment chart. Note: clinical outcomes: blood pressure, glycated haemoglobin, Body Mass Index and total cholesterol. 


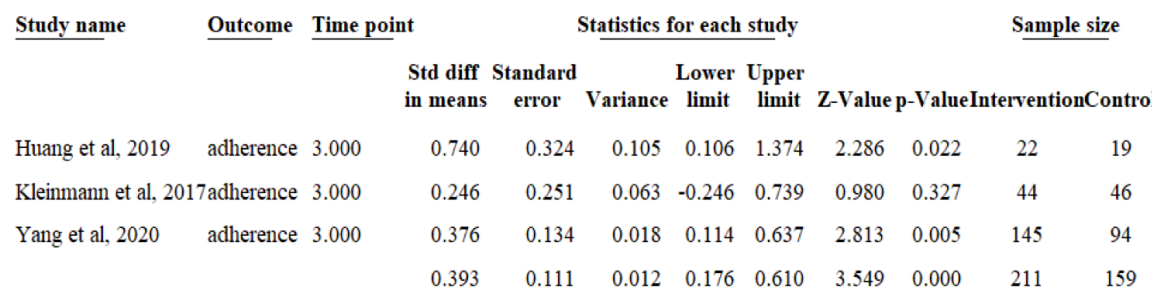

$\mathrm{Q}=1.507, \mathrm{df}=2$, p-value $0.471 R^{2}=0 \%$
Std diff in means and $95 \% \mathrm{CI}$

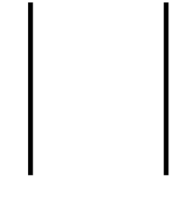

$-2.00$

$-1.00$

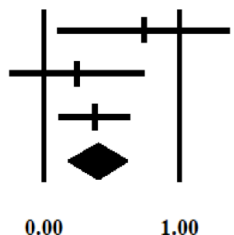

2.00

Favours Usual Care Favours Mobile app

Figure 3 Fixed-effects meta-analysis: standardized mean difference (SMD) of medication adherence at 3 months between diabetes self-management education and support app and usual care groups.

\section{Intervention effect}

Review estimates were aggregated from study results at 3 months from baseline measurements. Pooled analysis showed that DSMES app improved medication adherence ( $\mathrm{SMD}=0.393,95 \%$ CI 0.17 to $0.61 ; I^{2}=0 \%$ ) and patient adherence (SMD $=0.632,95 \%$ CI 0.17 to $1.094 ; I^{2}=83 \%$ ) compared with usual care. Interventions also reduced Hba1c (MD $=-0.314,95 \%$ CI -0.477 to $\left.-0.151 ; I^{2}=0 \%\right)$ and BMI (MD $=-0.28,95 \%$ CI -0.545 to $-0.015 ; I^{2}=0 \%$ ). The authors did not pool results for BP (few studies) and total cholesterol (high heterogeneity). Results are illustrated in figures 3-5.

\section{DISCUSSION}

After 3 months of DSMES app intervention, participants had a small but significant improvement in medication adherence compared with those receiving usual care. The intervention also had a small and moderate effect on HbA1c and BMI, respectively.

Medication adherence improved significantly after receiving 3 months of intervention. Intervention effect was also highly consistent among students $\left(I^{2}=0 \%\right)$. Improved medication adherence is a good development as it is associated with positive health outcomes and delay in development of DM complications. ${ }^{35}$ However, achieving optimal adherence is an ongoing challenge as patients are required to strictly follow dosages and recommendations from initiation to long-term continuation of disease management throughout their lives. ${ }^{36}$ The current review shows that DSMES apps could help by acting as reminders and communication channels, scheduling and calculating doses as well. In a way, they do unburden patients and allow them to sync DM management effortlessly into their lives. A review by Peng et al found similar findings in participants with various chronic diseases. ${ }^{18}$ The use of simple messages has been widely studied with contrasting results, ${ }^{10}{ }^{17}$ including the use of mobile apps to improve medication adherence.

Lifestyle adherence was measured in one study. ${ }^{32}$ Thus, a postanalysis outcome, patient adherence, was made by aggregating medication and lifestyle adherence outcomes. The pooled estimate showed a moderate and significant effect compared with usual care, though there was high heterogeneity observed. Adherence to lifestyle recommendations is important as they play a crucial role in controlling T2D. Patients who manage the diet and increase physical activity are more likely to fare well in terms of health outcomes. ${ }^{37}$ Lifestyle therapy adherence is also crucial to controlling other comorbidities such as hypertension, which were present in all studies. ${ }^{38} 39$

Studies all had cointerventions; most commonly, provider portals and independent reminders were used. These components illustrate the importance of patient-provider interaction, which have proven useful in improving other health outcome. ${ }^{40}$ The review does not have evidence that this feature, or any other intervention component or population characteristic, was vital in improving adherence partly due to failure to conduct moderator analysis.

\begin{tabular}{|c|c|c|c|c|c|c|c|c|c|c|c|}
\hline \multirow[t]{2}{*}{$\underline{\text { Study name }}$} & \multirow[t]{2}{*}{ Outcome } & \multirow[t]{2}{*}{ Time point } & \multicolumn{7}{|c|}{ Statistics for each study } & \multicolumn{2}{|c|}{ Sample size } \\
\hline & & & $\begin{array}{c}\text { Difference } \\
\text { in means }\end{array}$ & $\begin{array}{l}\text { Standard } \\
\text { error }\end{array}$ & Variance & $\begin{array}{c}\text { Lower } \\
\text { limit }\end{array}$ & $\begin{array}{c}\text { Upper } \\
\text { limit }\end{array}$ & Z-Value & p-Value & Intervention & Control \\
\hline Dominguez et al, 2019 & $\mathrm{HbAlc}$ & 3.000 & -0.100 & 0.167 & 0.028 & -0.427 & 0.227 & -0.600 & 0.548 & 94 & 91 \\
\hline Huang et al, 2019 & $\mathrm{HbAlc}$ & 3.000 & -0.500 & 0.660 & 0.436 & -1.795 & 0.795 & -0.757 & 0.449 & 22 & 19 \\
\hline Kleinmann et al, 2017 & $\mathrm{HbAlc}$ & 3.000 & -0.600 & 0.269 & 0.072 & -1.128 & -0.072 & -2.229 & 0.026 & 44 & 46 \\
\hline Yang et al, 2020 & $\mathrm{HbAlc}$ & 3.000 & -0.350 & 0.104 & 0.011 & -0.554 & -0.146 & -3.359 & 0.001 & 145 & 94 \\
\hline & & & -0.314 & 0.083 & 0.007 & -0.477 & -0.151 & -3.769 & 0.000 & 305 & 250 \\
\hline
\end{tabular}

$\mathrm{Q}=2.977, \mathrm{df}=3$, p-value $0.395{ }^{2}=0 \%$

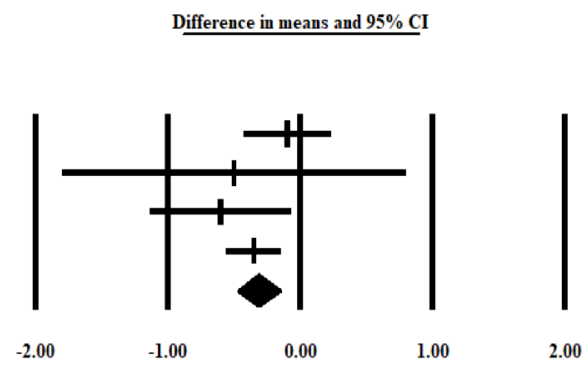

Favours Mobile app Favours Usual Care

Figure 4 Fixed-effects meta-analysis: mean difference (MD) of glycated haemoglobin at 3 months between diabetes selfmanagement education and support app and usual care groups. 


\begin{tabular}{|c|c|c|c|c|c|c|c|c|c|c|c|}
\hline \multirow[t]{2}{*}{ Study name } & \multirow[t]{2}{*}{ Outcome } & \multirow[t]{2}{*}{ Time point } & \multicolumn{7}{|c|}{ Statistics for each study } & \multicolumn{2}{|c|}{ Sample size } \\
\hline & & & $\begin{array}{c}\text { Difference } \\
\text { in means }\end{array}$ & $\begin{array}{c}\text { Standard } \\
\text { error }\end{array}$ & Variance & $\begin{array}{c}\text { Lower } \\
\text { limit }\end{array}$ & $\begin{array}{c}\text { Upper } \\
\text { limit }\end{array}$ & Z-Value & p-Value & Intervention & Control \\
\hline Dominguez et al, 2019 & BMI & 3.000 & -0.300 & 0.152 & 0.023 & -0.598 & -0.002 & -1.972 & 0.049 & 94 & 91 \\
\hline Huang et al, 2019 & BMI & 3.000 & -3.700 & 2.779 & 7.723 & -9.147 & 1.747 & -1.331 & 0.183 & 22 & 18 \\
\hline Kleinmann et al, 2017 & BMI & 3.000 & -1.100 & 1.080 & 1.166 & -3.217 & 1.017 & -1.019 & 0.308 & 44 & 46 \\
\hline Yang et al, 2020 & BMI & 3.000 & -0.090 & 0.307 & 0.095 & -0.693 & 0.513 & -0.293 & 0.770 & 145 & 94 \\
\hline & & & -0.280 & 0.135 & 0.018 & -0.545 & -0.015 & -2.072 & 0.038 & 305 & 249 \\
\hline
\end{tabular}

$\mathrm{Q}=2.490, \mathrm{df}=3$, p-value $0.477 R^{2}=0 \%$

\begin{abstract}
Figure 5 Fixed-effects meta-analysis: mean difference (MD) of Body Mass Index at 3 months between diabetes selfmanagement education and support app and usual care groups.
\end{abstract}

In terms of DSMES, with advances in technology, healthcare providers are also transitioning from or at least complimenting traditional delivery media with digital tools. ${ }^{14}$ As earlier stated, DSMES covers a lot of crucial topics including patient adherence to treatment. ${ }^{11}$ However, from the current review, studies show that mobile apps are mostly being used to offer self-management support or in combination with diabetes education. ${ }^{30-34}$ It is likely that pure diabetes education services are offered using either online or messaging services. Regardless, DSMES apps have the potential to offer both diabetes education and self-management support. ${ }^{32}$ Thus, implementers of DSMES interventions would ideally design a separate component of education using tools like websites and Short Message Service (SMS) or incorporate the education component into a self-management mobile app.

Our pooled effect estimate on HbAlc showed a small but significant reduction compared with usual care. The findings are in line previous literature. ${ }^{41}{ }^{42} \mathrm{HbAlc}$ is an important indicator of disease control though its efficacy as a screening tool is still debated. ${ }^{43}$ DSMES app functions, such as glucose monitoring and calculation of doses, would have played a role in improving $\mathrm{HbAlc}$ in review participants. In addition, medication adherence may have also influenced glycaemic control. ${ }^{44}$ Further research is required to shed more light on the association between HbAlc levels and medication adherence.

BMI is a direct indicator of patient adherence to lifestyle recommendations. It is noticeable that both patient adherence and BMI significantly improved, begging the question: how, if at all, are the two outcomes correlated? However, as earlier stated, the review was underpowered to conduct such analysis. BMI is crucial for patients with T2D, as increased BMI is an important risk factor in developing adverse cardiometabolic events and other noncommunicable diseases. ${ }^{3745}$ Managing BMI through lifestyle modifications should be given as much emphasis as medical treatment. Most treatment protocols, of course, include lifestyle management. ${ }^{46}$ However, few interventions targeted lifestyle therapies. ${ }^{32} 33$ On the other hand, studies that target lifestyle interventions seldom included patients with T2D. Consequently, most of these studies were excluded from the review.

\section{Applicability}

Intervention group participants were generally accepting of DSMES apps. Major advantages reported were convenience, ease of use, and being motivational and knowledgeable. Interventions acted as a medium of communication, increasing frequency of interactions from usual month or quarterly consultation to at time weekly. This advantage could possibly help providers spot anomalies faster, address emerging problems earlier and eventually reduce medical costs.

In terms of feasibility, first, for the system to work, there needs technical expertise, and second, patients are required to be digitally literate. The review's population was mostly old adults who might not be very technologically adept. ${ }^{18}$ However, studies reported assistance to participants whenever it was required.

\section{Strengths}

This review was tailored to the use of DSMES apps by patients with T2D and their impact on adherence. Results of all prespecified outcomes have shown a high consistency among included studies. Studies are all RCTs and were mostly conducted in PHC settings, which is generalisable to most patients with T2D.

\section{Limitations}

Several factors need to be taken into consideration. First, studies are mostly feasible trials. Second, few studies meant reviewers could not achieve moderator analysis. The first two limitations could indicate that relatively little research has been conducted on this topic. Third, it is difficult to ascertain which intervention component was vital in improving outcomes. Adherence was self-reported in some studies, raising the possibility of reporter bias. Fourth, use of theoretical frameworks was reported in only one study, ${ }^{33}$ although they are known to be important in development of interventions.

\section{Further research}

First, DSMES app intervention needs to be tested in larger trials. In addition, the review reports results measured at 3 months. There is a need to study the long-term impact of DSMES apps on both medication and lifestyle therapy 
adherence. Furthermore, long-term follow-up would allow the measurement of outcomes, such as persistence. Lastly, use of theoretical frameworks during intervention implementation is highly recommended in future studies. ${ }^{10}$

\section{CONCLUSION}

Pooled analysis showed that DSME apps had significant small to moderate effects on medication adherence, HbA1c and BMI of patients with T2D in comparison to usual care. All results had low heterogeneity. Participant feedback showed apps were reliable, easy to use and convenient, though most required to be phone literate. Evidence comes from feasibility trials with generally moderate risk of bias. Larger trials with longer follow-up periods using theory-based interventions are required to improved current evidence.

\section{Author affiliations}

${ }^{1}$ Nkhata Bay District Health Office, Nkhata Bay District Hospital, Nkhata Bay, Malawi ${ }^{2}$ Global Health and Development Department, College of Public Health, Taipei Medical University, Taipei, Taiwan

${ }^{3} \mathrm{PhD}$ Program in Nursing, College of Nursing, Taipei Medical University, Taipei, Taiwan

${ }^{4}$ Australian Institute of Health Service Management, Tasmanian School of Business and Economics, University of Tasmania, Sydney, New South Wales, Australia ${ }^{5}$ Graduate Institute of Biomedical Informatics, College of Medical Science and Technology, Taipei Medical University, Taipei, Taiwan

${ }^{6}$ International Center for Health Information Technology (ICHIT), Taipei Medical University, Taipei, Taiwan

${ }^{7}$ Department of Dermatology, Taipei Municipal Wan Fang Hospital, Taipei, Taiwan

${ }^{8}$ International Medical Informatics Association (IMIA), Geneva, Switzerland

${ }^{9}$ Research Center for Artificial Intelligence in Medicine, Taipei Medical University, Taipei, Taiwan

${ }^{10} \mathrm{PhD}$ Program of Global Health and Health Security, College of Public Health, Taipei Medical University, Taipei, Taiwan

\section{Twitter Dumisani Enricho Nkhoma @dnkhoma}

Contributors DEN and UI conceived the idea of the paper. DEN and CJS searched, screened articles and collected data. DEN and KJB did the quality assessment; DEN and CJS conducted the analysis, supervised by UI; and KJB, DEN and CJS did the paper writing, supervised and edited by KJB, DG, Y-CL and UI.

Funding This work was supported in part by Ministry of Science and Technology (MOST), Taiwan (ROC), under grant MOST109-2221-E-038-015.

Competing interests None declared.

Patient consent for publication Not required.

Ethics approval The review collected secondary information reported in published studies in which consent was already sort from study participants. Therefore, no ethical approval was required.

Provenance and peer review Not commissioned; externally peer reviewed.

Data availability statement № available data to share. The current systematic review and meta-analysis study used previous published research papers which have been cited in the manuscript.

Supplemental material This content has been supplied by the author(s). It has not been vetted by BMJ Publishing Group Limited (BMJ) and may not have been peer-reviewed. Any opinions or recommendations discussed are solely those of the author(s) and are not endorsed by BMJ. BMJ disclaims all liability and responsibility arising from any reliance placed on the content. Where the content includes any translated material, BMJ does not warrant the accuracy and reliability of the translations (including but not limited to local regulations, clinical guidelines, terminology, drug names and drug dosages), and is not responsible for any error and/or omissions arising from translation and adaptation or otherwise.
Open access This is an open access article distributed in accordance with the Creative Commons Attribution Non Commercial (CC BY-NC 4.0) license, which permits others to distribute, remix, adapt, build upon this work non-commercially, and license their derivative works on different terms, provided the original work is properly cited, appropriate credit is given, any changes made indicated, and the use is non-commercial. See: http://creativecommons.org/licenses/by-nc/4.0/.

\section{ORCID iDs}

Yu-Chuan (Jack) Li http://orcid.org/0000-0001-6497-4232

Usman lqbal http://orcid.org/0000-0002-0614-123X

\section{REFERENCES}

1 Kaiser AMYB, Zhang N, Der Pluijm WVAN. Global prevalence of type 2 diabetes over the next ten years (2018-2028). Diabetes 2018;67:202.

2 World Health Organization. Fact sheet: the top 10 causes of death. Geneva, Switzerland: World Health Organization, 2017.

3 Bommer C, Sagalova V, Heesemann E, et al. Global economic burden of diabetes in adults: projections from 2015 to 2030. Diabetes Care 2018:41:963-70.

4 World Health Organization. Global report on diabetes. Geneva: World Health Organization, 2016.

5 Asche C, LaFleur J, Conner C. A review of diabetes treatment adherence and the association with clinical and economic outcomes. Clin Ther 2011;33:74-109.

6 Lerman I. Adherence to treatment: the key for avoiding long-term complications of diabetes. Arch Med Res 2005;36:300-6.

7 Organization WH. Adherence to long-term therapies: evidence for action: World Health organization 2003.

8 Cramer JA. A systematic review of adherence with medications for diabetes. Diabetes Care 2004;27:1218-24.

9 Lam WY, Fresco P. Medication adherence measures: an overview. Biomed Res Int 2015;2015:1-12.

10 Farmer AJ, McSharry J, Rowbotham S, et al. Effects of interventions promoting monitoring of medication use and brief messaging on medication adherence for people with type 2 diabetes: a systematic review of randomized trials. Diabet Med 2016;33:565-79.

11 Beck J, Greenwood DA, Blanton L, et al. 2017 national standards for diabetes self-management education and support. Diabetes Educ 2018;44:35-50.

12 Dayer L, Heldenbrand S, Anderson P, et al. Smartphone medication adherence apps: potential benefits to patients and providers. J Am Pharm Assoc 2013;53:172-81.

13 World Health Organization. eHealth Geneva: World Health organization, 2019. Available: https://www.who.int/ehealth/en/ [Accessed 1 Apr 2019].

14 American Diabetes Association. 7. Diabetes Technology: Standards of Medical Care in Diabetes-2019. Diabetes Care 2019;42:S71-80.

15 Jahns R. Currently, only $1.2 \%$ of diabetics that have a smartphone use a diabetes app, 2018. Available: http://research2guidance com/currently-only-12-of-diabetics-that-have-a-smartphoneuse-a-diabetes-app-until-2018-the-share-will-rise-to-78-globally/ [Accessed 16 Aug 2015].

16 Adams A, Shankar M, Tecco H. 50 things we now know about digital health consumers. Rock Health 2016.

17 Thakkar J, Kurup R, Laba T-L, et al. Mobile telephone text messaging for medication adherence in chronic disease: a meta-analysis. JAMA Intern Med 2016;176:340-9.

18 Peng $\mathrm{Y}$, Wang $\mathrm{H}$, Fang Q, et al. Effectiveness of mobile applications on medication adherence in adults with chronic diseases: a systematic review and meta-analysis. J Manag Care Spec Pharm 2020;26:550-61.

19 Lunde P, Nilsson BB, Bergland A, et al. The effectiveness of smartphone apps for lifestyle improvement in noncommunicable diseases: systematic review and meta-analyses. J Med Internet Res 2018;20:e162.

20 Wu X, Guo X, Zhang Z. The efficacy of mobile phone Apps for lifestyle modification in diabetes: systematic review and metaanalysis. JMIR Mhealth Uhealth 2019;7:e12297.

21 Vermeire E, Wens J, Van Royen P, et al. Interventions for improving adherence to treatment recommendations in people with type 2 diabetes mellitus. Cochrane database of systematic reviews 2005.

22 Moher D, Liberati A, Tetzlaff J, et al. Preferred reporting items for systematic reviews and meta-analyses: the PRISMA statement. PLoS Med 2009;6:e1000097.

23 Cochrane Public Health. Review authors: cochrane collaboration, 2019. Available: https://ph.cochrane.org/review-authors 
24 Sterne JAC, Savović J, Page MJ, et al. RoB 2: a revised tool for assessing risk of bias in randomised trials 2019;366:14898.

25 Wan X, Wang W, Liu J, et al. Estimating the sample mean and standard deviation from the sample size, median, range and/or interquartile range. BMC Med Res Methodol 2014;14:135.

26 Hozo SP, Djulbegovic B, Hozo I. Estimating the mean and variance from the median, range, and the size of a sample. BMC Med Res Methodol 2005;5:13.

27 Higgins JP, Green S. Cochrane Handbook for systematic reviews of interventions. John Wiley \& Sons, 2011.

28 Higgins JPT, Thompson SG, Deeks JJ, et al. Measuring inconsistency in meta-analyses 2003;327:557-60.

29 Kardas P, Lewandowski K, Bromuri S. Type 2 diabetes patients benefit from the COMODITY12 mHealth system: results of a randomised trial. J Med Syst 2016;40:259.

30 Huang Z, Tan E, Lum E, et al. A smartphone APP to improve medication adherence in patients with type 2 diabetes in Asia: feasibility randomized controlled trial. JMIR Mhealth Uhealth 2019;7:e14914.

31 Yang Y, Lee EY, Kim H-S, et al. Effect of a mobile Phone-Based Glucose-Monitoring and feedback system for type 2 diabetes management in multiple primary care clinic settings: cluster randomized controlled trial. JMIR Mhealth Uhealth 2020:8:e16266.

32 Alonso-Domínguez R, García-Ortiz L, Patino-Alonso MC, et al. Effectiveness of a multifactorial intervention in increasing adherence to the Mediterranean diet among patients with diabetes mellitus type 2: a controlled and randomized study (EMID study). Nutrients 201910.3390/nu11010162. [Epub ahead of print: 14 Jan 2019].

33 Kleinman NJ, Shah A, Shah S, et al. Improved medication adherence and frequency of blood glucose Self-Testing using an $\mathrm{m}$-Health platform versus usual care in a multisite randomized clinical trial among people with type 2 diabetes in India. Telemed J E Health 2017;23:733-40.

34 Brath H, Morak J, Kästenbauer T, et al. Mobile health (mHealth) based medication adherence measurement - a pilot trial using electronic blisters in diabetes patients. Br J Clin Pharmacol 2013;76:47-55.

35 Krass I, Schieback P, Dhippayom T. Adherence to diabetes medication: a systematic review. Diabet Med 2015;32:725-37.
36 Vrijens B, De Geest S, Hughes DA, et al. A new taxonomy for describing and defining adherence to medications. Br J Clin Pharmacol 2012;73:691-705.

37 Klein S, Sheard NF, Pi-Sunyer X, et al. Weight management through lifestyle modification for the prevention and management of type 2 diabetes: rationale and strategies. A statement of the American diabetes association, the North American association for the study of obesity, and the American Society for clinical nutrition. Am J Clin Nutr 2004;80:257-63.

38 Castaneda C, Layne JE, Munoz-Orians L, et al. A randomized controlled trial of resistance exercise training to improve glycemic control in older adults with type 2 diabetes. Diabetes Care 2002;25:2335-41.

39 Esposito K, Maiorino MI, Bellastella G, et al. A journey into a Mediterranean diet and type 2 diabetes: a systematic review with meta-analyses. BMJ Open 2015;5:e008222.

40 Chatterjee S, Davies MJ, Heller S, et al. Diabetes structured selfmanagement education programmes: a narrative review and current innovations. Lancet Diabetes Endocrinol 2018;6:130-42.

41 Liu K, Xie Z, Or CK. Effectiveness of mobile App-Assisted self-care interventions for improving patient outcomes in type 2 diabetes and/ or hypertension: systematic review and meta-analysis of randomized controlled trials. JMIR Mhealth Uhealth 2020;8:e15779.

42 Hou C, Carter B, Hewitt J, et al. Do mobile phone applications improve glycemic control $(\mathrm{HbA} 1 \mathrm{c})$ in the self-management of diabetes? A systematic review, meta-analysis, and grade of 14 randomized trials. Diabetes Care 2016;39:2089-95.

43 Larese $\mathrm{J}$. When is hemoglobin A1c inaccurate in assessing glycemic control. NYU Langone internal medicine Blog, faculty peer reviewed 2012.

44 Wabe NT, Angamo MT, Hussein S. Medication adherence in diabetes mellitus and self management practices among type-2 diabetics in Ethiopia. N Am J Med Sci 2011;3:418-23.

45 Look AHEAD Research Group, Wing RR. Long-Term effects of a lifestyle intervention on weight and cardiovascular risk factors in individuals with type 2 diabetes mellitus: four-year results of the look ahead trial. Arch Intern Med 2010;170:1566-75.

46 American Diabetes Association. 4. Lifestyle Management: Standards of Medical Care in Diabetes-2018. Diabetes Care 2018;41:S38-50. 